\title{
p16 a biomarker of aging and tolerance for cancer therapy
}

\author{
Hyman B. Muss ${ }^{1}$, Andrew Smitherman ${ }^{2}$, William A. Wood ${ }^{1}$, Kirsten Nyrop ${ }^{1}$, Sascha Tuchman ${ }^{1}$, \\ Paramjeet K. Randhawa ${ }^{3}$, Amy R. Entwistle ${ }^{3}$, Natalia Mitin ${ }^{3}$, Shlomit S. Shachar ${ }^{4,5}$
}

${ }^{1}$ Department of Medicine, ${ }^{2}$ Department of Pediatrics, University of North Carolina School of Medicine and the Lineberger Comprehensive Cancer Center, Chapel Hill, NC, USA; ${ }^{3}$ Sapere Bio, Research Triangle Park, NC, USA; ${ }^{4}$ Ruth and Bruce Rappaport Faculty of Medicine at the Technion, Haifa, Israel; ${ }^{5}$ Department of Oncology, Rambam Health Care Campus, Haifa, Israel

Contributions: (I) Conception and design: All authors; (II) Administrative support: None; (III) Provision of study materials or patients: None; (IV) Collection and assembly of data: None; (V) Data analysis and interpretation: All authors; (VI) Manuscript writing: All authors; (VII) Final approval of manuscript: All authors.

Correspondence to: Hyman B. Muss, MD. Department of Medicine, University of North Carolina School of Medicine and the Lineberger Comprehensive Cancer Center, 170 Manning Drive, Campus box 7305, Chapel Hill, NC 27599, USA. Email: muss@med.unc.edu.

\begin{abstract}
There is great variability in life-expectancy, physical, cognitive, and functional domains in cancer patients of similar chronologic age. Nowhere is this more apparent than among middle-aged and older patients. However, even in younger patients of similar age, extensive exposure to environmental stressors can cause great variability in health status. A biomarker that would reflect biologic age and any and all health deficits in a cancer patient at a distinct point in time might help predict long term outcomes related to treatment, especially toxicity and overall survival. p16 ${ }^{\mathrm{INK} 4 \mathrm{a}}$ (hereafter referred to as p16) expression represents an ideal biomarker that reflects both cellular senescence and biologic aging. In murine models, p16 expression reflects biologic aging in almost all organs. Preliminary findings in patients with cancer support p16 measurement as a marker of physiologic aging and predictor of toxicity in patients treated with chemotherapy. This review describes the role of p16 in cell senescence, the methodology of p16 measurement in humans, preliminary studies of p16 in humans, and the potential clinical utility of p16 in guiding treatment for cancer patients.
\end{abstract}

Keywords: p16; biomarker; senescence; cancer; aging

Submitted Feb 10, 2020. Accepted for publication Mar 03, 2020.

doi: $10.21037 /$ tcr.2020.03.39

View this article at: http://dx.doi.org/10.21037/tcr.2020.03.39

\section{Introduction}

The world's population is aging and while in the $19^{\text {th }}$ century human life expectancy increased from 20 to 40 years, from the $19^{\text {th }}$ century to the $20^{\text {th }}$-century life expectancy doubled to 80 years and continues to increase (1). Globally, the population over age 65 years is the fastestgrowing age group. The US population continues to age and by 2050, the number of Americans aged 65 and older is projected to be 88.5 million, which is more than double the population of 40.2 million in 2010 (2). This aging of the population is associated with an increased cancer incidence which rises dramatically with age and suggests that between 2010 and 2030 , there will be a $67 \%$ increase in cancer incidence for patients age 65 years or older (3). Moreover, patients who are 65 years and older have an 11-fold risk of developing cancer (4). Further, increasing age portends a higher risk of multi-morbidity and frailty, complicating treatment choices for aging cancer patients and creating an urgent need to better understand the impact of aging in decision-making for cancer treatments.

Aging is a heterogeneous process and chronologic age is a poor reflector of molecular age, health risk, and life span. For clinical decision making in cancer, life expectancy is a key consideration, and patients of similar chronological ages but with major differences in life expectancy are likely to be offered different treatment options. High-quality tools are available to calculate life expectancy (5), and for older 
cancer patients specifically, clinical and geriatric assessment data have been used to develop models to predict surgical outcomes and chemotherapy toxicity $(6,7)$. Clinical judgment is usually accurate for assessing cancer treatment tolerance in extremely healthy or very frail patients but can be inaccurate for a large segment of middle-aged and older patients with moderate comorbidity or loss of function. Better tools are needed to facilitate treatment decisions in patients who may self-rate their health as good but who may actually have substantial functional and other deficits (8). Another concern is undertreatment, which frequently happens when using chronologic age alone to make cancer treatment decisions. For example, older adults with breast cancer are less likely to be offered chemotherapy (9-11) with $47 \%$ of women over 65 receiving chemotherapy compared to $81 \%$ of women aged 20 to 64 years (12). Even healthier older patients, with no or very few co-morbid illnesses (diabetes, kidney disease, or heart disease) are less likely to receive appropriate treatment (12). The National Cancer Institute has voiced significant concern over these treatment discrepancies, highlighting women over 65 years with early breast cancer as a "group at high risk for not receiving appropriate treatment" (12). Given that the average lifespan for women in the US is 81 years, many older women with potentially curable breast cancer can significantly benefit from adjuvant chemotherapy. There is a major need for accurate, biologically plausible, and clinically accessible biomarkers that would augment current clinical assessments used for treatment decision making in all cancer patients. The ideal biomarker would identify patients most likely to experience increased toxicities associated with accelerated aging or other deficits, thereby enabling preventive strategies that might minimize long term morbidity.

While age-related diseases have diverse phenotypes, there is increasing recognition of common biological underpinnings with cellular senescence as the nexuslinking subcellular changes due to genetic factors and environmental insults with lifespan and the eventual decline in health and development of frailty (13). Senescence is a state of permanent cellular growth arrest (replicative senescence); because senescent cells cannot undergo cell division, they cannot contribute to tissue repair and homeostasis. In addition, senescent cells secrete myriad proinflammatory cytokines, contributing to inflammation and impaired organ regeneration. Accumulation of senescent cells broadly contributes to tissue aging across organ systems and suggests that biomarkers of senescence could be used to identify a patient's "molecular age". Molecular age, in turn, could be used for better risk stratification to maximize treatment efficacy and minimize adverse events. This review focuses on p16, a crucial biomarker of cell senescence and molecular aging, the methodology of p16 measurement in humans, and the utility of p16 expression in guiding cancer treatments.

\section{Biomarkers of aging}

Studies over the last several years have identified molecular biomarkers that may serve as markers of the aging process. The American Federation of Aging Research (AFAR) has stated that a true biomarker of aging is one "that predicts a person's physiological, cognitive, and physical function in an age-related way, must be testable and not harmful to test subjects, and should work in laboratory animals as well as humans" (https://www.afar.org/docs/AFAR_ BIOMARKERS_OF_AGING_2016.pdf). Candidates for biomarkers of aging have been reviewed elsewhere (14-16). They include inflammatory markers characterized by a pervasive low-grade and chronic inflammation ('inflammaging') such as C-reactive protein (CRP), interleukins (most prominently IL-6) and tumor necrosis factors (TNF- $\alpha$ ) (17), telomere length (short telomeres trigger DNA damage checkpoints that cause mitotic arrest and cell senescence) (18), DNA methylation (19), measurement of maximal oxygen consumption (vO2max) (20), and sarcopenia (21). These biomarkers change throughout the lifespan, correlate with an increase in frailty and mortality, and can, in theory, be used to quantify the aging process. Leukocyte telomere length (LTL) decreases with increasing chronologic age and with activities known to accelerate aging such as cigarette smoking (22). Serum levels of D-Dimer also increase with age and are associated with clinical frailty (23). Likewise, the expression of IL-6 increases with aging and correlates with age-associated morbidity and mortality (24). While these biomarkers unequivocally change with age, none is well-suited for individual risk prediction due to several important technical and biological limitations. First, some of these markers (e.g., LTL) are challenging to measure and exhibit a very small dynamic range (e.g., <40\%) across the human lifespan, with substantial variation among individuals at baseline/birth $(25,26)$. Although LTL can be used to estimate aging in individuals with genetically short telomeres (dyskeratosis congenita and some idiopathic pulmonary fibrosis patients), it does not have clinical accuracy for predicting biological age in the general population $(24,27,28)$. Most aging-associated cytokines (e.g., IL-6) are highly 


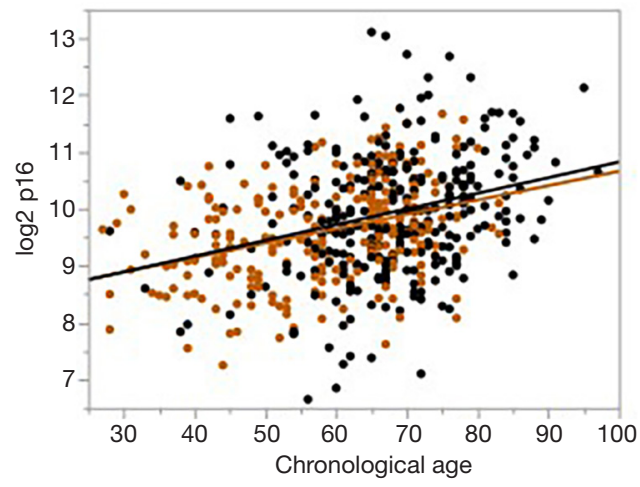

Figure 1 Chronologic age versus p16 expression in 536 patients, 242 with breast cancer prior to chemotherapy (orange dots) and 294 patients with heart disease (black dots). trend lines of breast cancer and heart patients are almost identical. Note that p16 expression rises dramatically with increasing age and that there is a wide variation in p16 expression for patients of similar age.

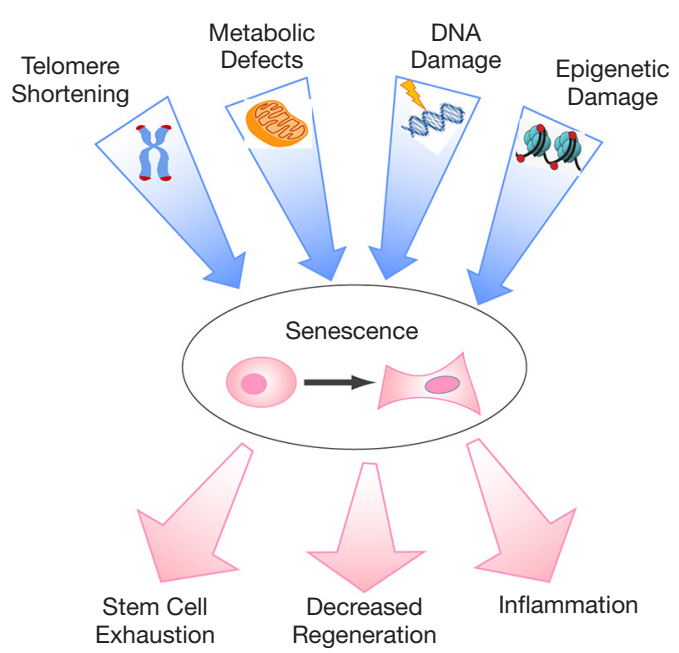

Figure 2 Senescence and its role in age-related decline. Accumulation of damage from environmental, genetic and lifestyle factors results in epigenetic changes, telomere shortening, DNA damage and mitochondrial dysfunction, all leading to induction and accumulation of senescent cells. Senescent cells, in turn, cause age-related decline by limiting stem cell and tissue regenerative capacity as well as increased inflammation. Adapted from $\mathrm{McHugh}$ and Gill [2018].

labile influenced by inflammation, with transient high expression observed in adults with acute infections and other inflammatory conditions. Signatures of cytokine panels, known as senescence-associated secretory phenotype (SASPs), are notoriously tissue-specific and require immediate sample processing to accurately capture in vivo levels. Recently, DNA methylation has been suggested as a marker of biological age. Although intriguing, all studies of DNA methylation have been powered to predict different endpoints, such as best fit with chronological age or mortality, but so far none has been shown to have clinical utility in any disease $(29,30)$.

The expression of p16 meets the AFAR criteria for an effective biomarker of aging. It dramatically increases with age and encodes for a protein that blocks cyclin-dependent kinase which promotes cellular senescence and permanent cell-cycle arrest (31). In addition to meeting the AFAR definition of an aging biomarker, measurement of p16 expression is a quantitative measurement of senescencea central mechanism by which environmental, genetic, and lifestyle damage affects the aging of an individual and leads to functional decline. In addition, and especially for patients receiving cancer chemotherapy, there is excellent evidence in children (32) and emerging evidence in adults $(33,34)$ that such treatment accelerates both physiologic and biologic aging. What is especially compelling about p16 expression as a biomarker of aging is its dramatic and dynamic range among patients of similar chronologic age. Figure 1 shows the wide range in p16 expression that can vary by several $\operatorname{logs}(\log 2)$ for cancer or heart disease patients of similar chronologic age. Similar changes have been shown in normal controls (31).

\section{Aging and senescence}

The depletion of senescent cells in experimental animal models demonstrates the critical role of senescence in agerelated declines in physical function as well as the initiation and progression of common, chronic diseases (Figure 2). Transplant of senescent cells from old animals into young animals induces similar age-related loss of function and shortened lifespan (35). Senolytic therapies (pharmacologic $v s$. transgenic depletion of senescent cells), in turn, restore function and increase lifespan in both normally aged mice and mice aged by transplanting senescent cells into young donor mice (35-37). Tissue-level analysis in animal models demonstrates that senescence occurs throughout the organism $(37,38)$. Moreover, senescent cells are highly metabolically active and are capable of inducing senescence in bystander cells at both local and distant sites $(35,39)$. Cumulatively, this evidence suggests that senescence has systemic effects that can be measured and may be regulated at the organismal level. Consequently, the role 


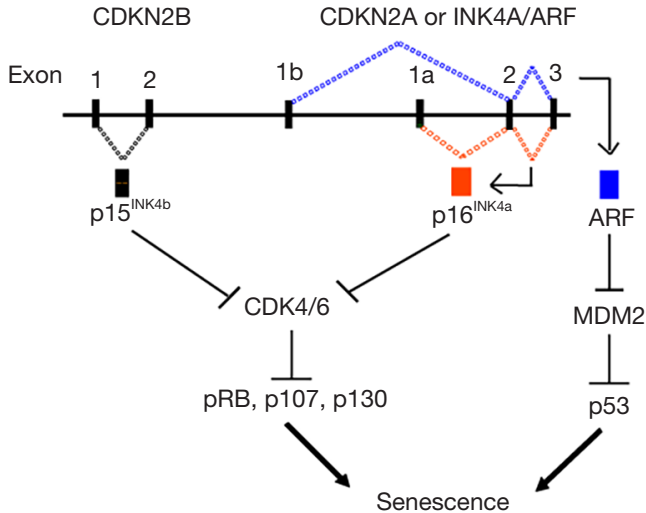

Figure 3 Genetics and signaling of p16/ARF locus. P16 and ARF mRNAs arise from the same locus but encode distinct peptides. p16 is encoded from exons 1a, 2, and 3 and ARF from exons 1b, 2, and 3. p16 is an inhibitor of cell-cycle dependent kinases CDK 4/6 and induces senescence through that pathway. ARF can also induce senescence but acts through the p53 pathway.

of senescence in human disease is an area of strong interest and active inquiry.

\section{p16 and senescence}

In humans, p16 is found on chromosome 9p21 in close proximity to two other tumor suppressor genes, p15 and ARF (Figure 3) (40). p16 and ARF genes are alternatively spliced from the same locus, but because their first exons are distinct, the resulting protein sequences are distinct. Signals that eventually lead to senescence upregulate p16 and p15 cell cycle genes. P16 is a cyclin-dependent kinase inhibitor that acts on CDK4/6 kinases to prevent phosphorylation of retinoblastoma $(\mathrm{Rb})$ family proteins and promotes $\mathrm{G} 1$ cell cycle arrest, leading to senescence. ARF promotes senescence through the p53 tumor suppression pathway. Expression of p16 is not detected in young cells but is potently activated by stress factors that promote cellular senescence (40-43). This results in a permanent arrest of cellular growth and maintenance of the p16 transcript which is required for maintenance of the senescent phenotype. Nearly all of the compelling animal data cited above, including evidence for a causal role for senescence in age-related diseases, has been generated using experimental models in which senescence is defined by expression of the p16 biomarker (35-37,44-49). p16 expression is highly dynamic, increasing exponentially with age in all mammalian species tested to date $(38,50,51)$. Thus, unlike the other biomarkers of aging described above, p16 plays a direct and essential role in the initiation and maintenance of senescence. Much of the early work pertaining to p16 was done in the laboratory of Dr. Ned Sharpless at the University of North Carolina (31,38,40,52-54). This work prompted the creation of p16-based senescence animal models (36,55-57) which have transformed our understanding of senescence as a critical aging mechanism and led to current research exploring the age-accelerating effects of medical procedures and therapeutics.

In humans, ample unbiased genome-wide association study data implicate p16 as a critical determinant of human aging and age-related conditions (52,58-61). Clinical studies addressing the significance of senescence in human frailty and aging are just beginning. A recent study demonstrated that p16 expression in biopsied intramuscular fat independently correlates with lower muscle strength and worse walking performance $(15,62)$, and small exploratory studies of senolytic therapies are being planned $(63,64)$. Consequently, the role of senescence in human disease is an area of strong interest and active inquiry with therapeutic implications for a rapidly aging society.

\section{Measurement of the p16 biomarker}

In order to study senescence in humans where multi-tissue analysis is not possible, the Sharpless lab developed an assay to measure p16 expression in an easily obtained and clinically relevant tissue-peripheral blood (31). Given the systemic nature of senescence, they postulated that measurement of senescence in the blood may serve as a surrogate for the organismal senescent load. First, they discovered that expression of p16 in peripheral blood mononuclear cells (PBMCs), frequently used to measure expression for other clinical diagnostics, is too low to allow reproducible detection. Therefore, they identified a blood subtype that expresses the majority of p16, T-cells-and used isolated T-cells to measure p16 expression. Second, they observed that the measurement of p16 mRNA is more reliable than its derived protein. p16 mRNA and its protein are both upregulated in senescent cells; however, p16 mRNA, with an unusually long half-life of greater than 24 hours (65-68), is significantly more stable than protein [halflife 30 minutes to 3.5 hours (69)]. Given this unparalleled stability, an RT-PCR assay measuring p16 expression can be used to measure senescence with analytical precision and reproducibility superior to other RT-PCR diagnostics.

Using this assay to measure p16 mRNA in T-cells, 
Sharpless and colleagues found that p16 was highly dynamic, doubling about every 8 years. As expected, expression of p16 was not detected in young adult donors (under 20 years of age). Subsequent studies demonstrated that expression of p16 in human T-cells is influenced by a variety of age-promoting stimuli, including cigarette smoking, physical inactivity (31), cytotoxic chemotherapy administration (34), chronic HIV infection (70), and bone marrow transplantation (33). Collectively, these data show that a blood-based diagnostic test of $p 16$ gene expression is a practical biomarker of molecular age, with expression in T-cells acting as a faithful reporter of senescence load.

More recently, Mitin and collaborators (Sapere Bio, Research Triangle Park, NC, USA) have continued to build on the research version of the p16 assay to develop a reproducible, sensitive and analytically validated version that can be easily used in a clinical setting. Investigators added an extensive panel of positive and negative controls isolated from human subjects, expanded the panel of housekeeping genes used to normalize p16 expression, and established quality control parameters to monitor every step of the assay from sample collection through p16 RTPCR measurement. Implementation of these steps allowed measurement of p16 mRNA with a coefficient of variation $<4 \%$ (Mitin et al., unpublished data).

Traditionally, T-cells were isolated within 6 to 8 hours of venipuncture to obtain the desired reproducibility/precision of p16 measurement. If not isolated within 8 hours, p16 expression levels drop below detection, especially in younger patients. The need to isolate T-cells the same day creates a major research challenge in clinical settings, especially in oncology practice where most patients are seen in community clinic settings. To address this challenge, the Mitin lab has developed a whole blood stabilization solution which, when added to the blood collection tube can preserve T-cells and corresponding p16 expression levels for up to 48 hours. This improvement allows for blood samples to be collected in the clinic and sent to a central lab for processing without compromising sample integrity and opens the way for investigators to further explore the role of senescence biomarkers in a wide variety of research settings with analytically and clinically validated methods.

\section{Clinical utility of p16 measurement in cancer}

To-date p16-based molecular age in clinical studies has been studied in two major ways. Because p16 expression varies greatly among patients of similar chronologic age, baseline p16 measures have been correlated with individual patient outcomes, illuminating otherwise unseen risk/ resilience prior to treatment. Second, measuring changes in p16 expression pre- and post-treatment have revealed differences in the degree to which chemotherapies and high-dose chemotherapy and stem cell transplants are ageaccelerating, potentially depleting physiologic reserve and increasing the risk of frailty and more rapid health decline in survivorship. Although impressive changes in p16 expression have been noted with chemotherapy (discussed below), the long-term implications of these changes on the development of chronic disease and life expectancy remain uncertain. Only long-term follow-up of patients in current studies will answer these questions. p16 measurement at baseline and after treatment may be used in the future to optimize treatment decisions for individual patients, where invisible molecular aging confers risk. Below, we summarize current studies demonstrating the age-accelerating effects of chemotherapy and the relationship of baseline p16 and the risk of specific chemotherapy-induced toxicities in hematologic and solid tumor malignancies. This work suggests that the knowledge of a patient's molecular age and understanding its clinical implications prior to treatment has the potential to improve treatment decisions.

\section{p16 in hematologic malignancy}

Acute myelogenous leukemia, non-Hodgkin's lymphoma, and multiple myeloma are malignancies that are agedependent, with the majority of patients diagnosed after age 65 (71) (https://seer.cancer.gov/csr/1975_2016/). Multiple myeloma is primarily treated with systemically administered medications including "novel agents" that are not designed to function via DNA-damaging mechanisms. However, high-dose chemotherapy (usually melphalan) with autologous stem cell transplantation remains a key standard of care for managing myeloma in some patients. Melphalan, an alkylator, functions by causing DNA crosslinking and mutagenesis (72). Consequently, investigations surrounding both natural aging and therapy-induced accelerated aging are highly relevant in multiple myeloma, including biomarkers of aging such as p16. Investigators at the Ohio State University compared peripheral blood T-cell p16 expression levels in normal controls $(\mathrm{n}=17)$ and patients with both newly diagnosed (treatment-naïve) and relapsed or treatment-refractory ( $\mathrm{n}=11$ and 24 respectively), with ages ranging from 35-82 across the entire cohort. After controlling for age, the authors observed greater p16 
expression in patients with relapsed/refractory myeloma $v s$. normal controls but p16 was not significantly elevated in newly diagnosed myeloma patients compared to controls. The study team then investigated the impact of specific therapies. "Immunomodulatory agents", primarily lenalidomide, did not appear to impact p16 levels. However, when p16 expression was measured in a small number of patients both before and after high-dose chemotherapy with autologous stem cell transplant, an increase in p16 expression was observed post-transplant that ranged from 2.25- to 32.2-fold. This magnitude of p16 increase was correlated with 33.7 years of natural aging (based on approximate "conversions" described elsewhere (31). The authors concluded that high-dose chemotherapy and stem cell transplant physiologically age a patient, whereas novel agents do not seem to do so (73).

A related analysis by members of our group examined patients undergoing autologous or allogeneic stem cell transplant for various hematologic malignancies (33). Of 63 patients, 15 (24\%) had myeloma, 14 of whom were treated with high-dose chemotherapy and autologous transplantation. Among these patients, no associations were found between pre-transplant chemotherapy (i.e., cytotoxic $v s$. novel agents) and pre-transplant p16 expression, but that population was relatively small and heterogeneous. In this same study, 14 patients had lymphoma (10 treated with autologous stem cells and 4 with allogeneic cells) and 19 had acute leukemia (all treated with allogeneic cells). Patients receiving autologous transplants had a 3.1-fold increase in p16 expression pre-to post-transplant that corresponded to 28 years of accelerated aging $(\mathrm{P}=0.002)$, compared to those treated with allotransplant (1.9-fold-increase or 16 years of accelerated aging, $\mathrm{P}=0.0004)$. For all patients who received autologous transplants, no baseline characteristics were associated with pre-transplant p16 expression. However, for patients who received allogeneic transplants, more chemotherapy cycles before transplant, a history of prior autologous transplantation, or a history of alkylating agent exposure, were all significantly associated with higher pretransplant p16 expression $(\mathrm{P}<0.01$ for each). These preto post-transplant changes in p16 are among the highest observed in the literature to date, as compared to the impact of other noxious immune stimuli such as non-transplant cytotoxic chemotherapy, HIV infection, or tobacco use. Further exploration of gene expression in T-cells in this study by gene set enrichment analysis (GSEA) suggested that transplant induced not only p16 expression but also a broader milieu of changes associated with immune aging manifested as a shift from central memory and naïve cells to effector cells and regulatory T-cells, changes that have been associated with aging in other studies (74).

Taken together, these limited data suggest that the development of multiple myeloma itself and treatment with non-DNA-damaging novel agents may not necessarily induce p16 expression and accelerated aging, while treatment with DNA-damaging agents such as highdose melphalan with autologous stem cell transplantation may induce aging. In patients with acute leukemia and lymphoma treated with an allogeneic transplant, more cycles of chemotherapy and alkylating agents increased p16 prior to transplant as compared to controls. Further studies of aging, p16 expression, hematologic malignancy are ongoing by our group and others.

\section{p16 and cancer treatment in children, adolescents, and young adults}

Improved care for childhood, adolescent, and young adult cancers has led to increased survival with more than an estimated 500,000 survivors (75). However, due to cancer and its treatment, many of these survivors' experience accelerated aging as evidenced by a disproportionate loss of exercise capacity (76), cognitive decline (77), and early development of chronic medical morbidities (32) leading to discordance between chronological and molecular ages. As has been observed in adults, limited data among childhood cancer survivors suggest that cancer treatment increases levels of p16 expression to induce senescence. A single study of ten patients treated with cranial radiation for childhood acute lymphoblastic leukemia ( 1 to 10 years old at diagnosis) compared p16 mRNA expression in paired skin biopsies from the scalp and buttock (78). The authors observed a nearly 6 -fold increase in p16 expression in the radiated scalp samples as compared to the buttock biopsies, suggesting increased senescence in response to ionizing radiation exposure. The survivors were on average 12 years post therapy, suggesting that the increase in senescence persists over time.

Further research is needed to better define p 16 expression in childhood, adolescent, and young adult cancer survivors and to understand the role that cellular senescence plays in the early aging phenomenon seen in this population. For example, is the aging trajectory similar in childhood cancer survivors and naturally aging adults after the initial chemotherapy-induced age acceleration? Or, will the damage accumulated during 


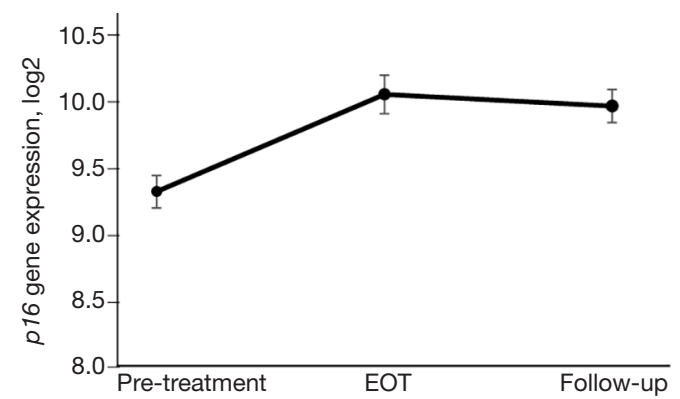

Figure 4 Doxorubicin-containing chemotherapy regimens are potent inducers of p16 gene expression. In this sample of 51 patients with early breast cancer, neoadjuvant or adjuvant doxorubicin chemotherapy increased $p 16$ gene expression by the EOT. All 51 patients had p16 assessed pre, EOT, and at followup. EOT-depending on the regimen treatment duration was on average 131 (range, 96-238) days. P16 expression remained elevated at the follow-up time point. Follow up sampling was on average 192 days from the EOT (range, 91-308 days). EOT, end of treatment.

chemotherapy continue to impact their aging trajectory and continue to accelerate aging? How long does it take for the chemotherapy-induced $\mathrm{p} 16$ boost to present itself as a clinical measurement of health decline and frailty? Can a post-chemotherapy rehabilitation program guided by p16-based risk stratification help slowdown progression to clinical frailty to extend a healthy lifespan in the survivors? Can p16 expression be used as a proximal biomarker to assess the efficacy of such rehabilitation interventions? Ongoing work of our group is currently attempting to answer these questions and help improve clinical decisionmaking for patients at risk for the potential development of treatment-related physiologic impairment and morbidities, and that may guide the frequency and type of monitoring during survivorship.

\section{p16 in solid tumors}

Higher baseline p16 expression prior to chemotherapy treatment in breast cancer patients has been shown to be associated with greater treatment-related fatigue (57) and a higher risk of chemotherapy-induced peripheral neuropathy (manuscript in preparation). In a study of women with breast cancer, changes in 16 in patients receiving adjuvant and neoadjuvant chemotherapy has been explored with initial findings showing a rapid, marked, and sustained increase from pre- to post-treatment, especially in patients receiving anthracycline-based chemotherapy (34). In that study, p16 measurement in peripheral blood T-cells was performed at four time points in a prospectively followed cohort of 33 women with stage I-III breast cancer. It was observed that 16 expression increased shortly after the start of chemotherapy and remained elevated 12 months later, with a $75 \%$ absolute increase in p16 expression that was equivalent to 14.7 years of chronologic aging. Expression of two senescence-associated cytokines, VEGFA and MCP1, was also significantly increased during chemotherapy, but LTL and IL-6 were not. In a cross-sectional cohort of 176 breast cancer survivors who were followed for a median of 3.4 years, the $39 \%$ of women treated with chemotherapy had an increase in p16 expression comparable to 10.4 years of chronological aging (34). A more recent study in a different cohort of women with early-stage breast cancer found that the largest increases in p16 levels were associated with anthracycline-based chemotherapy (Figure 4) as opposed to non-anthracycline chemotherapy (Muss et al., manuscript under review).

In a study of 42 patients age 70 and older with stage IV non-small cell lung cancer treated with nanoparticle albumin-bound-paclitaxel after previous treatment with a platinum doublet regimen, p16 was assessed at baseline and during treatment in 17 patients (79). As expected, baseline p16 in these heavily treated patients was higher than in agematched normal controls but and there was no convincing change of p16 during chemotherapy treatment suggesting that, unlike anthracyclines and alkylating agents, taxanes may have minimal effects on T-cell aging.

\section{Discussion}

Clinical decision-making for cancer patients is complicated by patient age, comorbidities, perceived treatment tolerance, and life expectancy. Thus, an area of intense investigation is the development of accurate, simple, clinically applicable biomarkers of molecular age that characterize not only a patient's ability to tolerate and benefit from therapy in the short-term but also reflect the age-accelerating effects of treatment that may lead to premature health decline and frailty. While efficacy will always be the predominant driver of treatment decisions in oncology, an accurate measure of toxicity risk becomes critical when: (I) there are multiple treatment options with similar efficacy but different toxicities, (II) when treatment benefit could be considered marginal relative to toxicity risk, and (III) when preventive measures are selectively applied to at-risk patients. Such 
scenarios, which are common in cancer treatment, require the oncologist and patient to make treatment decisions that could be much better informed with a clinically-validated biomarker of molecular age.

Measurement of molecular aging using p16 has great promise to provide robust information on outcomes and risk of toxicities associated with current cancer treatments. We believe that baseline p16 measurements will be confirmed by further research to predict the risks of treatment-related toxicity, chronic disease, and life-expectancy in cancer patients. Data from several clinical settings support the idea that measurement of p16 may be used to grade the aging effect of various chemotherapy regimens. Studies are currently in progress evaluating p16 as an independent predictor of regimen-specific chemotherapy-induced toxicities. In other areas of medicine, p16 expression is the focus of intensive research as a predictor of the success of solid organ transplant (80), chronic disease, and organ injury. Collectively, this work suggests that the knowledge of a patient's molecular age and its clinical implications prior to a treatment or procedure has the potential to improve treatment decisions and allows clinicians to employ targeted interventions to minimize a patient's risk and thereby improve outcomes.

\section{Acknowledgments}

Funding: We thank the Breast Cancer Research Foundation, New York, NY, USA; the Kay Yow Cancer Fund, Cary, NC, USA; the University Cancer Research Fund, University of North Carolina, Chapel Hill, NC, USA; and R01CA203023, National Cancer Institute for their support of our research.

\section{Footnote}

Provenance and peer review: This article was commissioned by the Guest Editor (Nathan A. Berger) for the series "Energy Balance, Aging, Obesity, and Cancer" published in Translational Cancer Research. The article was sent for external peer review organized by the Guest Editor and editorial office.

Conflicts of Interest: All authors have completed the ICMJE uniform disclosure form (available at http://dx.doi. org/10.21037/tcr.2020.03.39). The series "Energy Balance, Aging, Obesity, and Cancer" was commissioned by the editorial office without any funding or sponsorship. The authors have no other conflicts of interest to declare.

Ethical Statement: The authors are accountable for all aspects of the work in ensuring that questions related to the accuracy or integrity of any part of the work are appropriately investigated and resolved.

Open Access Statement: This is an Open Access article distributed in accordance with the Creative Commons Attribution-NonCommercial-NoDerivs 4.0 International License (CC BY-NC-ND 4.0), which permits the noncommercial replication and distribution of the article with the strict proviso that no changes or edits are made and the original work is properly cited (including links to both the formal publication through the relevant DOI and the license). See: https://creativecommons.org/ licenses/by-nc-nd/4.0/.

\section{References}

1. Estapé T. Cancer in the elderly: challenges and barriers. Asia Pac J Oncol Nurs 2018;5:40-2.

2. Vincent GK, Velkoff VA. The next four decades: the older population in the United States: 2010 to 2050. Washington DC: US Department of Commerce, Economics and Statistics Administration, US Census Bureau, 2010.

3. Smith BD, Smith GL, Hurria A, et al. Future of cancer incidence in the United States: burdens upon an aging, changing nation. J Clin Oncol 2009;27:2758-65.

4. Yancik R. Cancer burden in the aged: an epidemiologic and demographic overview. Cancer 1997;80:1273-83.

5. Yourman LC, Lee SJ, Schonberg MA, et al. Prognostic indices for older adults: a systematic review. JAMA 2012;307:182-92.

6. Hurria A, Mohile S, Gajra A, et al. Validation of a prediction tool for chemotherapy toxicity in older adults with cancer. J Clin Oncol 2016;34:2366-71.

7. Extermann M, Boler I, Reich RR, et al. Predicting the risk of chemotherapy toxicity in older patients: the Chemotherapy Risk Assessment Scale for High-Age Patients (CRASH) score. Cancer 2012;118:3377-86.

8. Jolly TA, Deal AM, Nyrop KA, et al. Geriatric assessmentidentified deficits in older cancer patients with normal performance status. Oncologist 2015;20:379-85.

9. Harlan LC, Clegg LX, Abrams J, et al. Community-based use of chemotherapy and hormonal therapy for early-stage breast cancer: 1987-2000. J Clin Oncol 2006;24:872-7.

10. DeMichele A, Putt M, Zhang Y, et al. Older age predicts 
a decline in adjuvant chemotherapy recommendations for patients with breast carcinoma: evidence from a tertiary care cohort of chemotherapy-eligible patients. Cancer 2003;97:2150-9.

11. Koroukian SM, Bakaki PM, Raghavan D. Survival disparities by Medicaid status: an analysis of 8 cancers. Cancer 2012;118:4271-9.

12. US National Institutes of Health. Cancer trends progress report-2011/2012 update. Bethesda: National Cancer Institute, 2012.

13. Calcinotto A, Kohli J, Zagato E, et al. Cellular senescence: aging, cancer, and injury. Physiol Rev 2019;99:1047-78.

14. Hubbard JM, Cohen HJ, Muss HB. Incorporating biomarkers into cancer and aging research. J Clin Oncol 2014;32:2611-6.

15. Ferrucci L, Gonzalez-Freire M, Fabbri E, et al. Measuring biological aging in humans: a quest. Aging Cell 2020;19:e13080.

16. Campisi J, Kapahi P, Lithgow GJ, et al. From discoveries in ageing research to therapeutics for healthy ageing. Nature 2019;571:183-92.

17. Franceschi C, Campisi J. Chronic inflammation (inflammaging) and its potential contribution to ageassociated diseases. J Gerontol A Biol Sci Med Sci 2014;69 Suppl 1:S4-9.

18. Sharpless NE, DePinho RA. Telomeres, stem cells, senescence, and cancer. J Clin Invest 2004;113:160-8.

19. Lu AT, Quach A, Wilson JG, et al. DNA methylation GrimAge strongly predicts lifespan and healthspan. Aging (Albany NY) 2019;11:303-27.

20. Jones LW, Courneya KS, Mackey JR, et al. Cardiopulmonary function and age-related decline across the breast cancer survivorship continuum. J Clin Oncol 2012;30:2530-7.

21. Shachar SS, Williams GR, Muss HB, et al. Prognostic value of sarcopenia in adults with solid tumours: a meta-analysis and systematic review. Eur J Cancer 2016;57:58-67.

22. Astuti Y, Wardhana A, Watkins J, et al. Cigarette smoking and telomere length: a systematic review of 84 studies and meta-analysis. Environ Res 2017;158:480-9.

23. Walston J, McBurnie MA, Newman A, et al. Frailty and activation of the inflammation and coagulation systems with and without clinical comorbidities: results from the Cardiovascular Health Study. Arch Intern Med 2002;162:2333-41.

24. Alter BP, Rosenberg PS, Giri N, et al. Telomere length is associated with disease severity and declines with age in dyskeratosis congenita. Haematologica 2012;97:353-9.
25. Valdes AM, Andrew T, Gardner JP, et al. Obesity, cigarette smoking, and telomere length in women. Lancet 2005;366:662-4.

26. Müezzinler A, Zaineddin AK, Brenner H. A systematic review of leukocyte telomere length and age in adults. Ageing Res Rev 2013;12:509-19.

27. Aubert G, Hills M, Lansdorp PM. Telomere length measurement-caveats and a critical assessment of the available technologies and tools. Mutat Res 2012;730:59-67.

28. Armanios M. Telomerase and idiopathic pulmonary fibrosis. Mutat Res 2012;730:52-8.

29. Chen BH, Marioni RE, Colicino E, et al. DNA methylation-based measures of biological age: metaanalysis predicting time to death. Aging (Albany NY) 2016;8:1844-65.

30. Horvath S, Raj K. DNA methylation-based biomarkers and the epigenetic clock theory of ageing. Nat Rev Genet 2018;19:371-84.

31. Liu Y, Sanoff HK, Cho H, et al. Expression of p16(INK4a) in peripheral blood T-cells is a biomarker of human aging. Aging Cell 2009;8:439-48.

32. Bhatia S, Armenian SH, Armstrong GT, et al. Collaborative research in childhood cancer survivorship: the current landscape. J Clin Oncol 2015;33:3055-64.

33. Wood WA, Krishnamurthy J, Mitin N, et al. Chemotherapy and stem cell transplantation increase p16INK4a expression, a biomarker of T-cell aging. EBioMedicine 2016;11:227-38.

34. Sanoff HK, Deal AM, Krishnamurthy J, et al. Effect of cytotoxic chemotherapy on markers of molecular age in patients with breast cancer. J Natl Cancer Inst 2014;106:dju057.

35. Xu M, Pirtskhalava T, Farr JN, et al. Senolytics improve physical function and increase lifespan in old age. Nat Med 2018;24:1246-56.

36. Baker DJ, Wijshake T, Tchkonia T, et al. Clearance of p16Ink4a-positive senescent cells delays ageing-associated disorders. Nature 2011;479:232-6.

37. Baker DJ, Childs BG, Durik M, et al. Naturally occurring p16(Ink4a)-positive cells shorten healthy lifespan. Nature 2016;530:184-9.

38. Krishnamurthy J, Torrice C, Ramsey MR, et al. Ink4a/ Arf expression is a biomarker of aging. J Clin Invest 2004;114:1299-307.

39. da Silva PFL, Ogrodnik M, Kucheryavenko O, et al. The bystander effect contributes to the accumulation of senescent cells in vivo. Aging Cell 2019;18:e12848.

40. Kim WY, Sharpless NE. The regulation of INK4/ARF in 
cancer and aging. Cell 2006;127:265-75.

41. Song Z, von Figura G, Liu Y, et al. Lifestyle impacts on the aging-associated expression of biomarkers of DNA damage and telomere dysfunction in human blood. Aging Cell 2010;9:607-15.

42. Brenner RJ, Pfaff JM. Mammographic features after conservation therapy for malignant breast disease: serial findings standardized by regression analysis. AJR Am J Roentgenol 1996;167:171-8.

43. te Poele RH, Okorokov AL, Jardine L, Cummings J, Joel SP. DNA damage is able to induce senescence in tumor cells in vitro and in vivo. Cancer Res 2002;62:1876-83.

44. Childs BG, Baker DJ, Wijshake T, et al. Senescent intimal foam cells are deleterious at all stages of atherosclerosis. Science. 2016;354:472-7.

45. Ogrodnik M, Miwa S, Tchkonia T, et al. Cellular senescence drives age-dependent hepatic steatosis. Nat Commun 2017;8:15691.

46. Jeon OH, Kim C, Laberge RM, et al. Local clearance of senescent cells attenuates the development of posttraumatic osteoarthritis and creates a pro-regenerative environment. Nat Med 2017;23:775-81.

47. Schafer MJ, White TA, Iijima K, et al. Cellular senescence mediates fibrotic pulmonary disease. Nat Commun 2017;8:14532.

48. Farr JN, Xu M, Weivoda MM, et al. Corrigendum: Targeting cellular senescence prevents age-related bone loss in mice. Nat Med 2017;23:1384.

49. Chinta SJ, Woods G, Demaria M, et al. Cellular senescence is induced by the environmental neurotoxin paraquat and contributes to neuropathology linked to Parkinson's disease. Cell Rep 2018;22:930-40.

50. Zindy F, Quelle DE, Roussel MF, et al. Expression of the p16INK4a tumor suppressor versus other INK4 family members during mouse development and aging. Oncogene 1997;15:203-11.

51. Melk A, Schmidt BM, Takeuchi O, et al. Expression of p16INK4a and other cell cycle regulator and senescence associated genes in aging human kidney. Kidney Int 2004;65:510-20.

52. Sharpless NE, DePinho RA. How stem cells age and why this makes us grow old. Nat Rev Mol Cell Biol 2007;8:703-13.

53. Tsygankov D, Liu Y, Sanoff HK, et al. A quantitative model for age-dependent expression of the p16INK4a tumor suppressor. Proc Natl Acad Sci U S A 2009; 106:16562-7.

54. Sorrentino JA, Krishnamurthy J, Tilley S, et al.
p16INK4a reporter mice reveal age-promoting effects of environmental toxicants. J Clin Invest 2014;124:169-73.

55. Burd CE, Sorrentino JA, Clark KS, et al. Monitoring tumorigenesis and senescence in vivo with a p16(INK4a)luciferase model. Cell 2013;152:340-51.

56. Ovadya Y, Krizhanovsky V. Strategies targeting cellular senescence. J Clin Invest 2018;128:1247-54.

57. Demaria M, O'Leary MN, Chang J, et al. Cellular senescence promotes adverse effects of chemotherapy and cancer relapse. Cancer Discov 2017;7:165-76.

58. Jeck WR, Siebold AP, Sharpless NE. Review: a metaanalysis of GWAS and age-associated diseases. Aging Cell 2012;11:727-31.

59. Melzer D, Frayling TM, Murray A, et al. A common variant of the p16(INK4a) genetic region is associated with physical function in older people. Mech Ageing Dev 2007;128:370-7.

60. Helgadottir A, Thorleifsson G, Manolescu A, et al. A common variant on chromosome 9p21 affects the risk of myocardial infarction. Science 2007;316:1491-3.

61. McPherson R, Pertsemlidis A, Kavaslar N, et al. A common allele on chromosome 9 associated with coronary heart disease. Science 2007;316:1488-91.

62. Justice JN, Gregory H, Tchkonia T, et al. Cellular senescence biomarker p16INK4a+ cell burden in thigh adipose is associated with poor physical function in older women. J Gerontol A Biol Sci Med Sci 2018;73:939-45.

63. Justice JN, Nambiar AM, Tchkonia T, et al. Senolytics in idiopathic pulmonary fibrosis: results from a firstin-human, open-label, pilot study. EBioMedicine 2019;40:554-63.

64. Justice JN, Niedernhofer L, Robbins PD, et al. Development of clinical trials to extend healthy lifespan. Cardiovasc Endocrinol Metab 2018;7:80-3.

65. Wang Y, Klijn JG, Zhang Y, et al. Gene-expression profiles to predict distant metastasis of lymph-node-negative primary breast cancer. Lancet 2005;365:671-9.

66. Hara E, Smith R, Parry D, et al. Regulation of p16CDKN2 expression and its implications for cell immortalization and senescence. Mol Cell Biol 1996;16:859-67.

67. Albain KS, Green SR, Lichter AS, et al. Influence of patient characteristics, socioeconomic factors, geography, and systemic risk on the use of breast-sparing treatment in women enrolled in adjuvant breast cancer studies: an analysis of two intergroup trials. J Clin Oncol 1996;14:3009-17.

68. Li J, Poi MJ, Tsai MD. Regulatory mechanisms of tumor suppressor P16(INK4A) and their relevance to cancer. 
Biochemistry 2011;50:5566-82.

69. Gombart AF, Yang R, Campbell MJ, et al. Inhibition of growth of human leukemia cell lines by retrovirally expressed wild-type p16INK4A. Leukemia 1997;11:1673-80.

70. Nelson JA, Krishnamurthy J, Menezes P, et al. Expression of p16(INK4a) as a biomarker of T-cell aging in HIVinfected patients prior to and during antiretroviral therapy. Aging Cell 2012;11:916-8.

71. Howlader N, Noone AM, Krapcho M, et al. SEER Cancer Statistics Review, 1975-2016. Bethesda: National Cancer Institute, 2019.

72. Samuels BL, Bitran JD. High-dose intravenous melphalan: a review. J Clin Oncol 1995;13:1786-99.

73. Rosko A, Hofmeister C, Benson D, et al. Autologous hematopoietic stem cell transplant induces the molecular aging of T-cells in multiple myeloma. Bone Marrow Transplant 2015;50:1379-81.

74. Desai A, Grolleau-Julius A, Yung R. Leukocyte function in the aging immune system. J Leukoc Biol 2010;87:1001-9.

75. Robison LL, Hudson MM. Survivors of childhood and

Cite this article as: Muss HB, Smitherman A, Wood WA, Nyrop K, Tuchman S, Randhawa PK, Entwistle AR, Mitin N, Shachar SS. p16 a biomarker of aging and tolerance for cancer therapy. Transl Cancer Res 2020;9(9):5732-5742. doi: 10.21037/ tcr.2020.03.39 adolescent cancer: life-long risks and responsibilities. Nat Rev Cancer 2014;14:61-70.

76. van Brussel M, Takken T, Lucia A, et al. Is physical fitness decreased in survivors of childhood leukemia? A systematic review. Leukemia 2005;19:13-7.

77. Krull KR, Sabin ND, Reddick WE, et al. Neurocognitive function and CNS integrity in adult survivors of childhood hodgkin lymphoma. J Clin Oncol 2012;30:3618-24.

78. Marcoux S, Le ON, Langlois-Pelletier C, et al. Expression of the senescence marker p16INK4a in skin biopsies of acute lymphoblastic leukemia survivors: a pilot study. Radiat Oncol 2013;8:252.

79. Weiss JM, Pennell N, Deal AM, et al. Nab-paclitaxel in older patients with non-small cell lung cancer who have developed disease progression after platinum-based doublet chemotherapy. Cancer 2020;126:1060-7.

80. van Willigenburg H, de Keizer PLJ, de Bruin RWF. Cellular senescence as a therapeutic target to improve renal transplantation outcome. Pharmacol Res 2018;130:322-30. 